\title{
Projet de recherche. Migration contrainte et Shoah. Réfugiés juifs en Europe occidentale 1938-1944
}

\author{
Insa Meinen et Ahlrich Meyer
}

\section{(2) OpenEdition \\ 1 Journals}

Édition électronique

URL : http://journals.openedition.org/cmc/606

DOI : $10.4000 /$ cmc.606

ISSN : 2684-3080

\section{Éditeur}

Fondation de la Mémoire Contemporaine

\section{Édition imprimée}

Date de publication : 1 décembre 2010

ISSN : 1377-1256

\section{Référence électronique}

Insa Meinen et Ahlrich Meyer, «Projet de recherche. Migration contrainte et Shoah. Réfugiés juifs en Europe occidentale 1938-1944 », Les Cahiers de la Mémoire Contemporaine [En ligne], 9 | 2010, mis en ligne le 01 février 2020, consulté le 17 novembre 2020. URL : http://journals.openedition.org/cmc/606 ; DOI : https://doi.org/10.4000/cmc.606 


\title{
Projet de recherche \\ Migration contrainte et Shoah \\ Réfugiés juifs en Europe occidentale 1938-1944
}

\author{
Insa Meinen - Ahlrich Meyer
}

Tel est l'intitulé d'un projet de recherche historique que nous menons avec l'appui de la Deutsche Forschungsgemeinschaft (DFG) depuis juillet 2009 à l'Université Carl von Ossietzky d'Oldenburg (RFA). Nous pouvons en l'occurrence compter sur la collaboration de la Fondation de la Mémoire contemporaine près le Centre interdisciplinaire d'Étude des Religions et de la Laïcité de l'Université libre de Bruxelles (CIERLULB). D'autres collaborateurs belges impliqués sont le Service des Victimes de la Guerre près le Service fédéral Sécurité sociale (SVG) et le Musée juif de la Déportation et de la Résistance de Malines (MJDR) ${ }^{1}$.

La persécution des Juifs en Europe occidentale est marquée par un paradoxe. Après que le régime national-socialiste eut expulsé par la force d'Allemagne et d'Autriche la majorité des Juifs, près de la moitié de ceux qui, jusqu'au début de la Seconde Guerre mondiale, avaient pu fuir aux Pays-Bas, en Belgique ou en France (approximativement 60.000 personnes) furent, au moment de l'occupation allemande de l'Europe occidentale, à nouveau traqués, appréhendés, internés à grand renfort de police et - avec bon nombre de Juifs locaux - amenés dans les camps d'extermination de l'Est. Avec le début des déportations d'Europe occidentale à l'été 1942 commença donc à l'intérieur des territoires contrôlés par les Allemands une nouvelle fuite, massive, de Juifs des Pays-Bas, de Belgique et de la France occupée, dont l'objectif premier était à présent de gagner la zone Sud, non encore occupée, de la France. C'est à peine si ces deux états de choses ont à ce jour fait l'objet de recherches historiques.

Leur préhistoire se trouve pour partie décrite dans les ouvrages de référence relatifs à l'émigration des Juifs d'Allemagne nazie ainsi que dans une série d'études à l'échelle nationale. L'histoire des réfugiés juifs en provenance du territoire du Reich allemand et d'Autriche a été

1 Traduit de l'allemand par Jacques Déom. 
jusqu'ici principalement traitée par la classique recherche portant sur l'émigration et l'exil ou dans le cadre de synthèses consacrées à la politique des réfugiés appliquée par des États susceptibles de les accueillir ${ }^{2}$. On mit alors surtout l'accent sur les années trente. L'intérêt dominant se portait également sur les artistes, écrivains, savants et opposants antifascistes, et donc sur les personnalités en vue de l'exil. C'est à peine si l'on vit dans les nombreux réfugiés anonymes des « sujets » de l'histoire. Ce n'est que tout récemment qu'a crû l'intérêt pour «l'exil des humbles ». Dès le début des années 80, le chercheur américain Herbert A. Strauss avait proposé une synthèse d'ensemble fondamentale d'orientation socio-statistique ${ }^{3}$. Mais les chiffres fournis par Strauss sur base de documents statistiques (60.000 réfugiés juifs en Europe occidentale, dont 30.000 victimes de la déportation) ne fournissent guère que des estimations, qu'il y aurait lieu de vérifier sur la base de sources plus larges et, au besoin, de corriger.

La littérature historique n'a envisagé le thème « affirmation de soi et résistance » des Juifs qu'en relation avec des structures organisées, à savoir dans le contexte des efforts de sauvetage dus à des organisations et cuvres d'assistance juives ou comme aspect des différents mouvements européens de résistance à l'occupation allemande. À quoi s'ajoute - ce qui est largement conditionné par la transmission de témoignages - que ce sont les survivants et les rescapés qui occupent l'avant de la scène, de même que les aides et les sauveurs, et non les victimes de la Shoah, dont les nazis cherchèrent à faire disparaître les traces ${ }^{4}$. Cela étant, la fuite s'avère un phénomène social de masse, une réaction collective à la persécution et à la menace d'extermination, parmi les «stratégies individuelles de survie » (Annie Kriegel) les plus importantes de l'époque nazie. L'option en faveur de la fuite autorise de surcroît des conclusions indirectes quant à ce que la population juive pressentait ou savait de l'objectif réel des déportations.

${ }^{2}$ Pour la Belgique, voir en particulier F. Caestecker, Ongewenste gasten. Joodse vluchtelingen en migranten in de dertiger jaren, Bruxelles, 1993.

${ }^{3}$ H. A. Strauss, « Jewish Emigration from Germany. Nazi policies and Jewish responses », dans Leo Baeck Institute Year Book, 25, 1980, pp. 313-361 ; 26, 1981, pp. 343-409.

${ }^{4}$ En ce qui concerne les aides, lire: J.-Ph. Schreiber, « L’accueil des réfugiés juifs du Reich en Belgique. Mars 1933 - septembre 1939: le Comité d'Aide et d'Assistance aux Victimes de l'Antisémitisme en Allemagne ", dans Les Cahiers de la Mémoire contemporaine, n 3, 2001, pp. 23 71 . 
Peu de recherches ont néanmoins été entreprises sur l'exode des Juifs au-delà des frontières. Quelques études régionales portent sur la fuite et sur l'aide qui lui fut apportée, par exemple dans les régions frontalières germano-néerlandaise ou germano-belge. On ne dispose pas de travaux sur l'implication de la douane ou de la police des frontières allemandes en Europe occidentale occupée. Une publication d'histoire sociale et économique consacrée à la ligne de démarcation française est venue combler une lacune de la recherche dans la mesure où l'on y traite de manière exhaustive des fuites de la zone Nord occupée vers le Midi de la France, qui échappe à l'occupation ${ }^{5}$. (Cette réalité reste dès lors en dehors des perspectives de notre projet, tout autant que les fuites via la frontière suisse et l'itinéraire qui mène des réfugiés de France en Espagne, à propos desquels on dispose à chaque fois de publications.) Par contre, le fait qu'à l'époque des déportations de masse entre 1942 et 1944 des Juifs cherchaient encore à fuir les PaysBas et la Belgique pour gagner la France n'a pour ainsi dire pas été pris en considération à ce jour par la recherche.

Enfin, notre propos rejoint les préoccupations de la recherche internationale sur la Shoah en Europe occidentale. La déportation des Juifs de France, de Belgique et des Pays-Bas est relativement bien étudiée et documentée. Dans les trois pays, on dispose à ce sujet d'une littérature scientifique considérable, qu'il est d'ailleurs de moins en moins possible de maîtriser. Il est par contre frappant que la recherche allemande récente sur la Shoah ne se soit, à quelques exceptions près, guère préoccupée de la persécution des Juifs en Europe occidentale. Dès lors, il est souhaitable d'examiner la «solution finale de la question juive » dans les divers pays européens dans des études comparatives et, dans ce cadre, d'intégrer surtout aussi l'Europe occidentale.

Une recherche portant sur les modalités de la fuite des Juifs en réaction à la persécution et à la déportation au plan supranational n'existe toujours pas. Pour ouvrir la voie à pareille recherche, nous nous concentrons en premier lieu sur deux groupes de personnes, à savoir : les Juifs d'Allemagne et d'Autriche qui trouvèrent refuge après

${ }^{5}$ E. Alary, La Ligne de démarcation, 1940-1944, Paris, 2003. 
1938 en Belgique et les Juifs des Pays-Bas et de Belgique qui, durant les années 1941-42 à 1944 cherchèrent à parvenir en France.

Plus précisément, les lignes directrices et questionnements suivants ont orienté notre enquête :

1. Nous étudions les déplacements de Juifs d'Allemagne et d'Autriche après 1938 et les fuites à l'intérieur de l'Europe occidentale occupée par les Allemands, placés sous le signe d'un génocide imminent. Ces flux se distinguent fondamentalement de l'émigration des Juifs du territoire du Reich dans les années qui ont suivi 1933. Notre sujet n'est pas l'exil et la perte de la terre natale. Nous admettons qu'un nouveau "type social de réfugié » s'est constitué après 1938, dont la migration forcée doit être envisagée sur fond de radicalisation de la persécution des Juifs et de la Shoah.

2. Notre projet de recherche à visée supranationale met l'accent sur la persécution des Juifs et la Shoah en Europe occidentale, en mettant en avant la Belgique comme pays d'accueil de réfugiés juifs en provenance d'Allemagne et d'Autriche. On sait peu, du moins en Allemagne, que la Belgique, avec son territoire exigu et densément peuplé, fut sans doute dans les années 1938-1939, nonobstant une politique d'asile qui se distinguait à peine de l'appareil de mesures dissuasives adopté par d'autres États européens, le plus important pays d'accueil de Juifs persécutés d'Allemagne et d'Autriche. Nous étudions de plus pour la première fois la fuite de Juifs des Pays-Bas vers la Belgique ou la France et de Belgique vers la France après la publication de l'interdiction d'émigration d'Europe occidentale (mai 1941) et durant la période de la déportation de masse de 1942 à 1944. À en juger par nos recherches préliminaires, l'envergure de ces fuites était bien plus considérable qu'on ne l'avait pensé jusqu'ici.

3. Nous ne nous limitons pas à la nationalité, mais choisissons comme critère le dernier domicile ; dès lors ce ne sont pas seulement des Juifs de nationalité allemande et ex-autrichienne qui sont pris en compte dans l'enquête, mais aussi les Juifs apatrides, entre autres polonais, qui vivaient en Allemagne et en Autriche. On s'en est tenu jusqu'ici dans la définition des groupes de réfugiés d'Allemagne à la nationalité, ce qui se comprend pour des raisons de commodité de la recherche, mais ne fait pas droit à la spécificité des groupes en question. Le problème traverse toute la littérature sur le sujet, au 
point que font par exemple défaut dans le récent mémorial des archives fédérales (Opfer der Verfolgung der Juden unter der nationalsozialistischen Gewaltherrschaft in Deutschland 1933-1945, Coblence, 2006) les noms de nombreux Juifs nés en Allemagne et déportés d'Europe occidentale, mais non détenteurs de la nationalité allemande. Pareils critères ne tiennent pas compte de l'histoire transnationale de la persécution de la population juive en Europe et conduisent à estomper méthodiquement la connexion entre migration contrainte et Shoah. Un coup d'œil sur la Belgique montre la dimension du problème : moins de $10 \%$ des Juifs enregistrés sous l'occupation allemande y détenaient la nationalité belge. C'est pourquoi nous retenons aussi le dernier domicile comme critère dans la recherche sur les fuites des Pays-Bas et de Belgique vers la France à partir de 1941.

4. Centrales sont les stratégies individuelles de survie et les efforts pour sauver leur vie des nombreux réfugiés juifs « anonymes », dont on relève les traces dans quantité de documents et de registres dispersés. Notre recherche ne part pas du témoignage des survivants, mais de ceux qui furent les victimes de la politique d'extermination nationalsocialiste. Comment et dans quelle mesure les victimes de la Shoah, sur la réaction défensive desquelles nous n'avons la plupart du temps qu'une bien maigre information, cherchèrent-elles à se mettre à l'abri de la déportation?

5. Notre recherche - en ce sens exemplaire - doit tirer au clair l'importance de la proportion des réfugiés juifs parmi les victimes de la déportation par rapport au nombre des victimes issues de la population juive " autochtone » d'Europe occidentale. Nous tenterons de plus d'apprécier les modalités de la fuite des Juifs par rapport à d'autres facteurs déterminants de la «solution finale» (forme de l'occupation allemande, disposition à collaborer des autorités nationales, comportement de la population non juive). Les différents chiffres des victimes en Europe occidentale (Pays-Bas 75\%, Belgique $44 \%$, France $25 \%$ de la population juive globale) sont-ils liés également aux mouvements de fuite des Juifs?

Pour de premiers résultats du projet, on verra : Ahlrich MeyerInsa Meinen, «Transitland Belgien. Jüdische Flüchtlinge in Westeuropa während der Zeit der Deportationen 1942 », dans Theresienstädter 
Studien und Dokumente, 2007, pp. 378-431. Trad. tchèque : "Belgie jako tranzitní země. Židovští uprchlíci v západní Evropě v době deportací roku 1942 », dans Terezínské studie a dokumenty, 2007, pp. 305-353. Trad. française : «La Belgique, pays de transit. Juifs fugitifs en Europe occidentale au temps des déportations de 1942 », dans Cahiers d'Histoire du Temps présent, $\mathrm{n}^{\circ}$ 20, 2008, pp. 145-194. 